\title{
Optical Layer Monitoring in Passive Optical Networks (PONs): A Review
}

\author{
Kivilcim Yuksel, Véronique Moeyaert, Marc Wuilpart, and Patrice Mégret \\ Faculty of Engineering, Mons, Boulevard Dolez 31, 7000, Mons, Belgium \\ Tel: (+3265) 37.43.51,Fax: (+3265) 37.41.99, e-mail: kivilcim.yuksel@fpms.ac.be
}

\begin{abstract}
Motivations, challenges and requirements of optical layer monitoring in PONs are discussed. An exhaustive review of monitoring systems is given considering both Time-Division Multiplexed PON (TDM-PON) and Wavelength-Division Multiplexed PON (WDM-PON) cases.
\end{abstract}

Keywords: Fiber-To-The-Home, optical layer monitoring, OTDR, Passive Optical Networks.

\section{INTRODUCTION}

Recently, installation of Fiber-to-the-x (FTTx, $\mathrm{x}=\mathrm{H}$ for home, B for building, $\mathrm{C}$ for curb and $\mathrm{N}$ for node) technology has started to accelerate as a strong alternative to the existing broadband access technologies based on copper pairs (Digital Subscriber Line, DSL) and coax (cable modems). This worldwide acceleration is largely due to both the considerable decrease in capital expenses (CAPEX) of introducing FTTx connectivity and its "future proof" nature in providing ever increasing user bandwidth requirements. The passive optical network (PON), one among several architectures that can be used in FTTx networks, is today the main choice of operators [1]. In a PON, there are only passive elements between the Optical Line Terminal (OLT) located in the central office $(\mathrm{CO})$ and the customer premises. A single optical fiber carries all traffic to a remote node $(\mathrm{RN})$, where there is a split by a passive optical power splitter into separate fibers that run to the individual Optical Network Units (ONU) or Optical Network Terminations (ONT). Such architecture is expected to lower the operation-and-maintenance expenses (OPEX) since there are no electronic components which are more prone to failure in the PON outside plant (OSP). There is no need for the operators to provide and monitor electrical power or maintain back-up batteries in the field [2]. Even though PONs have relatively low OPEX compared to active solutions, there is still room for the operators to save significant amount of OPEX using effective preventive maintenance of the physical infrastructure. In today's PON systems, the physical infrastructure is usually not entirely visible to the Network Management System (NMS). As a direct consequence, a physical failure can not be detected before creating service outage in upper layers which in turn may lead to tremendous loss in business for the operators. We can mention not only direct financial losses due to service interruption but also indirect financial losses due to bad reputation. These arguments have been gaining importance as the warranty on the quality of the infrastructure becomes a deciding factor in the strongly competitive marketplace.

The technical trends in new optical access technologies can be listed as service integration (voice, high-speed data and video), implementation of wavelength division multiplexing (WDM), high bit-rates (10 Gbit/s) and longer reach/higher split options [3], [4]. They all give rise to capacity increase and make a network failure more disastrous. The aim of preventive maintenance is to detect any kind of deterioration in the network that can cause suspended services and to localize these faults in order to avoid specially trained people deployed with dedicated and often expensive equipments for troubleshooting. PON infrastructure does not only suffer from accidental damage and environmental effects (e.g. water penetration in splice closures) but are also subject to a lot of changes after the network is installed and activated. As an example, the optical access network may not be initially fully loaded; subscribers would be turned up, possibly over an extended period of time [5]. Hence, network operator should continuously be aware if a change noticed by its monitoring system is service oriented or indeed a fault. In addition to that, it is crucial to discriminate the faults (accidental interruptions) from attacks (intentional interruptions) results in a strengthing of relations between optical maintenance functions and the security management.

All arguments mentioned above mean that the existing maintenance methods need to be updated making the monitoring in PONs an active research area. The number of scientific publications has significantly increased in the last a few years. Authors propose different approaches to take up the challenges of PON monitoring.

Ideal optical monitoring framework in PONs has the following general requirements:

- It should provide continuous, remote, automatic, and cost effective supervision of the physical layer.

- It should provide rapid and accurate detection of performance degradation as well as service disruption.

- It should unambiguously provide failure source location.

- $\quad$ The testing should not affect normal data transmission (non-intrusive testing).

- It should distinguish between a failure in the end-users' own equipment and a failure in the operator's network. Monitoring results should be conveyed to the NMS and evaluated here in detail enabling preventive countermeasures (like protection and restoration, isolation of attacking port...).

- It should be interoperable with many network variants (bitrate, protocol etc.). 
This paper gives an exhaustive literature review of monitoring systems for PONs. Section 2 discusses the challenges of the monitoring systems. Sections 3, 4 and 5 mainly categorize all the monitoring systems into three groups: solutions for TDM-PONs, solutions for WDM-PONs, and other solutions that are not based on conventional time domain reflectometry techniques and can be in principle applicable to both (TDM-, and WDM-) PON cases.

\section{CHALLENGES OF AUTOMATIC MONITORING SYSTEMS}

The most common maintenance tool employed for troubleshooting in long-haul, point-to-point fiber optic links is an Optical Time Domain Reflectometer (OTDR). However, implementation of OTDRs into PONs brings some testing challenges.

Firstly, fiber lines in PONs have many connection points with only short distances between them. It requires the use of an OTDR test equipment optimized for high resolution and short dead zones. Another consideration is that PONs contain passive branching components with high insertion losses. This requires high dynamic range (DR) at the OTDR. At the time being, it is difficult to ensure both high resolution and high DR capabilities for classical time domain reflectometry techniques.

Secondly, due to the large number of users, the measurement time may become another issue. If an OTDR trace is required for each user, assuming a CO with 16 OLT ports, each serving 32 users (totally $16 \times 32=512$ users), typical 3 minutes averaging time means about one day to monitor the whole network [6].

Beside these general considerations, another fundamental diagnostic challenge which is specific to TDM-PONs is known as point-to-multipoint problem. In the TDM-PON, the OTDR pulses launched into the fibre are passively split and propagate simultaneously in every branch after the splitting point. As a result, the backreflected and backscattered light signals from each branch add up together to form a "global" or composite trace that makes the interpretation of the OTDR trace from the CO a difficult task. Looking at the global trace without any additional information, one can not identify the faulty branch.

The fault quantization is another problem; in order to determine the "real loss" or "real return loss" of a fault, some further calculations are needed based on the network's parameters such as power levels measured during installation, splitting ratio, splitting insertion losses, ... [7], [8].

When it comes to WDM-PONs, guarantying the reliability in these networks might be even more crucial for the operators as the first expected aim of WDM-PONs is to transport high capacity services to business customers. The WDM-PON monitoring approaches suggest diagnostic requirements which are different from that of classical TDM-PONs. A conventional OTDR which operates at a single wavelength is not suitable to probe branches beyond the wavelength selective component at the RN of a WDM-PON.

Fig. 1 summarizes the different trends in PONs monitoring that have been published in the scientific literature. The remaining of this paper is based on this proposal of classification.

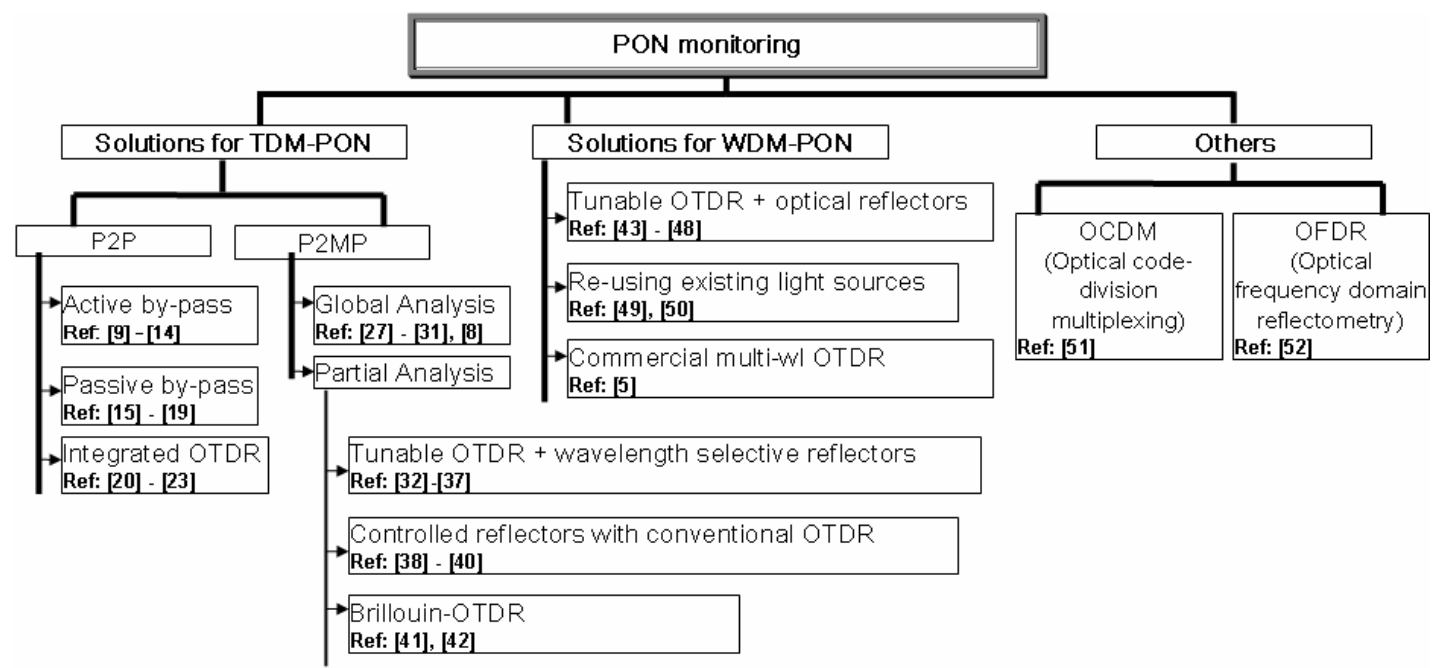

Figure 1. Classification of PON monitoring solutions.

\section{SOLUTIONS FOR TDM-PONS}

Solutions proposed for TDM-PONs can be further divided into two sub-groups; namely, point-to-point (P2P) monitoring approach and point-to-multipoint (P2MP) monitoring approach. 


\subsection{Point-to-Point Monitoring}

The key for P2P monitoring approach is to monitor all the branches individually either by the way of by-passing the splitters or by using embedded-OTDR functionality integrated into optical transceiver modules that are present at the ONU/ONT. By-pass of the splitting components can be realized by utilizing active equipments (active by-pass) or passive components (passive by-pass).

The main advantage of the solutions in this group is that the problems related to P2MP trace analysis are avoided. As a result, quantification and localization of the detected fault is an easy task.

\subsubsection{Active by-pass}

The active by-pass of the splitter can be performed by using optical selectors as shown in Fig. 2a. Depending on the PON topology (i.e. centralized, distributed ...) by-passed branching devices can be located in the CO, or at the RN. It should be noted that both cases are represented in the same figure (Fig. 2a) for the sake of simplicity. Utilization of reflectors in the end of branches (not shown in the Fig. 2a) eases the fault detection process by checking the presence and the height of the end reflection peak for the branch in test [9]. Active by-pass solution has been successfully implemented and deployed by NTT Japan in the automatic fiber line testing system called AURORA [10] and its extensions [11] where the by-passed branching devices are located in the central office. When the branching devices are in the outside plant, the problem of controlling and powering the optical selectors should be solved [12]. Recently, dynamic splitters which are remotely controlled through the optical fiber link have been reported, that might facilitate the by-pass of the splitter [13]. WDMs or wavelength insensitive couplers (WINC) could be used to introduce the monitoring signal into a fiber line. Alternatively, an optical waveguide coupler with embedded multilayered dielectric filters integrated to the splitter output was reported [14]. This approach using planar lightwave circuit (PLC) technology aims at reducing optical connection losses.

\subsubsection{Passive by-pass}

As shown in Fig. 2b, each branch is assigned to its own monitoring wavelength controlled by the tunable OTDR, while communication light is distributed to all branches uniformly. The by-pass of the splitters is performed by using wavelength mux/demux. Thus no active electronic devices (no powering) are required at the OSP [15], [16].

More than one branch may be assigned to one monitoring wavelength if the tuning range of the tunable OTDR is not sufficient to monitor a whole network with high splitting ratios [17]. Utilization of reflectors in the end of branches might ease the monitoring of each branch. For this purpose, dichroic reflective optical (DRO) filter that can be embedded in the fiber as well as Fiber Bragg gratings (FBG) were proposed [18]. Key components (tunable OTDR and routing WDM) of this solution are expensive to deploy in access networks [19].

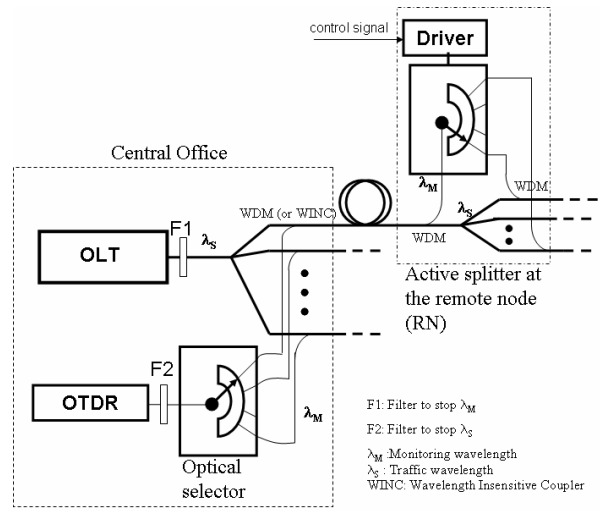

(a)

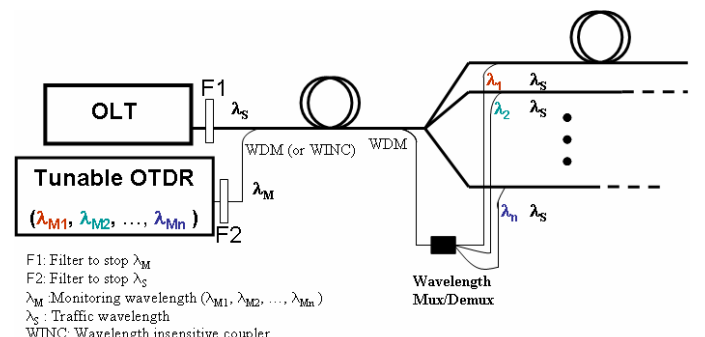

(b)

Figure 2. a) Active by-pass. WDMs or wavelength insensitive couplers (WINC) can be used to introduce the monitoring signal into a fiber line, b) Principle of passive by-pass.

\subsubsection{Integrated OTDR functionality}

The idea behind this solution is to measure the backreflected and backscattered echoes caused by the light signal transmitted upstream from already existing laser located inside ONU/ONT [20], [21]. Thus, the wavelength used for the monitoring is the same as for normal upstream data transmission and the need of connecting separate test equipments to the network is eliminated. 
The light signals used for OTDR excitations can be optical pulses as in the conventional OTDR technique [22] (pulse OTDR approach). In another approach, data bursts transmitted for the upstream data traffic are used as test signals by implementing a novel technique called 'negative-step OTDR' [23]. Monitoring should be performed during traffic interruptions for the former approach whereas the latter does not suffer from this problem. As the monitoring functionality is not centralized, reporting of the monitoring results is difficult in case of a broken fiber [22].

\subsection{Point-to-Multipoint Monitoring}

In P2MP monitoring approach, the OTDR is used directly on the whole PON from the OLT side without bypassing the splitters. In-service monitoring can be provided by using dedicated monitoring wavelength(s) different from the traffic wavelengths [24]. For this purpose, 1625-1650nm wavelength region is commonly considered [25], [26].

\subsubsection{Global analysis}

The One of the simple ways of making sense of "global" OTDR trace is to compare the current trace with a reference trace generally taken during the network installation or service activation [27]. When all branches are equipped with reference reflectors, the presence and qualitative height variations of reference reflection peaks help identifying the faulty branch as shown in Fig. 3a. Fault quantization is possible by additional calculation [8], [28]. Multiple faults reporting is also possible.

In principle, the evolution of the difference between two traces (reference and current) indicates the position of the fault. But when the splitting ratio increases, the change created by the fault on the global OTDR trace would be masked by the other branches. For instance, after a 1 by 16 splitter, even a fiber break could be invisible on a standard global OTDR trace. To solve this problem, a prototype of a high dynamic range OTDR was proposed [29]. In global analysis monitoring, system spatial resolution has a huge importance for two cases:

- if the distance between subscribers are small, hence discrimination between two end reflection peaks is difficult;

- when there is a high Fresnel reflection in the field which creates dead zones masking the faults.

For the first case where the reflection peaks coming from close users superimpose, a supplementary software analysis using neural network was claimed to improve the spatial resolution [30]. Another software analysis method was proposed for the second problematic case. This is called dead-zone-free signal analysis and is based on the utilization of a secondary OTDR 'ghost' signal to obtain a virtual bi-directional OTDR trace analysis [31].

\subsubsection{Use of tunable OTDR and wavelength selective reflectors}

In this partial analysis, each branch is successively identified using both a tunable OTDR and a wavelength selective reflector set at the end of each branch. For each wavelength of the tunable OTDR, all branches contribute to the backscattered signal but only one branch contributes to the end reflection peak [32] (Fig. 3b).

As tunable OTDR are not commercially available and are complex device, alternatives were proposed to obtain monitoring wavelengths [33]-[36].

FBGs and DROs might be used as reference reflectors. The so called wavelength coded tag (WCT) was also proposed [37]. A WCT can reflect a unique narrow-band signal within the monitoring band and passes all other wavelengths. Therefore it can be embedded not only in the end of the branches but also at the output ports of the splitters in the distributed PON topology.

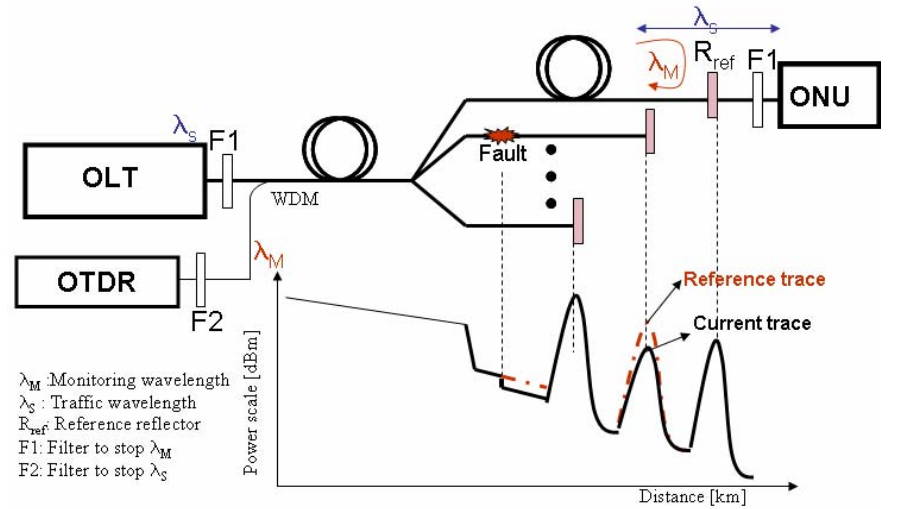

(a)

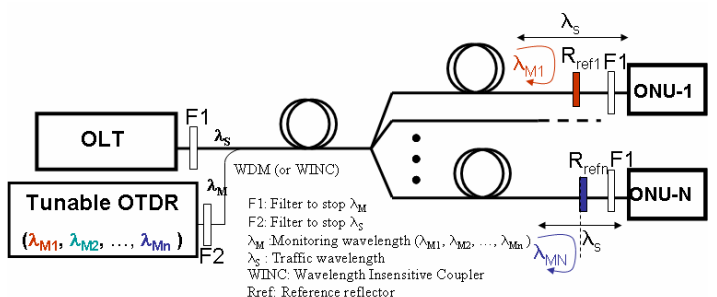

(b)

Figure 3. a) Global analysis, b) Monitoring system using tunable OTDR and reference reflectors. Filters placed at the ONU side are all different in terms of wavelength.

\subsubsection{Use of controlled reference reflections with conventional OTDR}

In this partial analysis, ONUs/OLTs are equipped with switchable reflective elements (SRE). For each OTDR measurement, only one subscriber is reflective and unambiguously identified. The system is shown in Fig. 4a. 
The SRE is a colorless device whose design is compatible with planar technologies, and thus mass production. During the monitoring, the SREs are successively remotely switched on and off from the CO: there is therefore only one peak at a time in the OTDR trace. The system is capable of localizing and quantifying several faults successively in the same branch or in different branches, even if they occur at the same distance [38].

The reflective element is remotely controlled by a coded signal at the monitoring wavelength sent downstream from the $\mathrm{CO}$ through the transmission line. The resolution in terms of fault detection and quantization is function of the dynamic range (DR) of the OTDR, the splitting ratio of the PON and the attenuation in each branch [39]. The signal to noise ratio after the splitter significantly degrades with the increase of splitting ratio. As a result, less faults can be detected. To alleviate this problem, the increase of the dynamic range of the OTDR by the way of Raman amplification was proposed [40].

\subsubsection{Use of Brillouin OTDR (BOTDR)}

This method needs a Brillouin-OTDR in the $\mathrm{CO}$ and fibers with individually assigned Brillouin frequency shifts (BFS), which are called identification fibers [41]. A unique BFS is assigned to each identification fiber by controlling the dopant concentration of the fiber core during fabrication process. A BOTDR basically consists in launching optical pulses in the fibers with a sufficient peak power and in recording the Brillouin backscattered signal.

To monitor a PON, a test pulse with a frequency $v$ is launched from a BOTDR (Fig. 4b). The pulse in each branch is scattered by the Brillouin effect. Branches are distinguished from each other by the frequency of the peak in the spectrum of all Brillouin scattered light. Once a fault is detected at an individual frequency (e.g. $v 1$ as represented in Fig. 4b), the BOTDR trace measured at that specific frequency ( $v 1)$ gives the location of fault in the corresponding branch (branch 1 in Fig. 4b) [42].

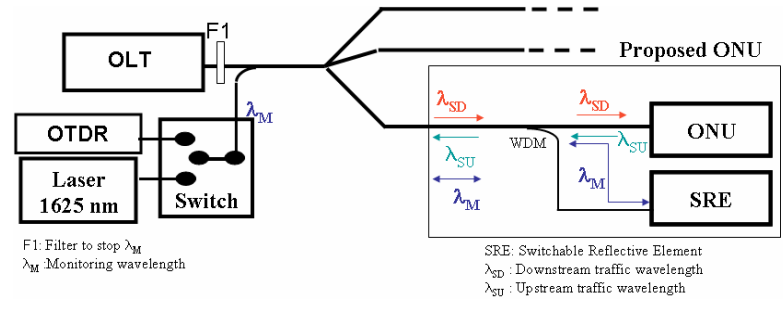

(a)

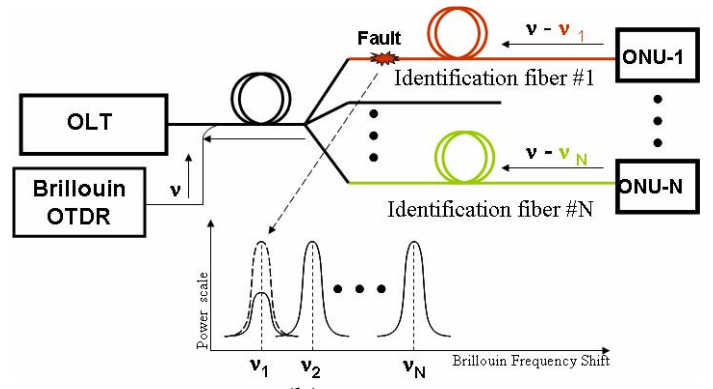

(b)

Figure 4. a) Monitoring system with SRE and conventional OTDR. A communication protocol is necessary between CO and SRE, b) Monitoring system with individually designed Brillouin frequency shifts and BrillouinOTDR [42].

\section{SOLUTIONS FOR WDM-PONs}

WDM-PONs typically use arrayed-waveguide grating (AWG) in the remote node (RN). The point-to-multipoint problem previously highlighted is inherently solved in this group because the monitoring wavelength is directed to only one branch after the splitter.

\subsection{Use of tunable OTDR or multi-wavelength source and optical reflectors}

The approach explained in paragraph 3.2.2 is readily applicable to WDM-PONs. Each branch is assigned a monitoring wavelength thanks to the already existing presence of the wavelength mux/demux device in the remote node $(\mathrm{RN})$ [43]. Having the same operational principle in mind, research has been devoted to some new interrogation techniques specific to WDM-PONs. Instead of using tunable OTDR, a broadband light source (BLS) is spectrally sliced at the RN into multiple monitoring channels which are reflected back to the CO by wavelength dependent optical reflectors at each ONU. Power spectral density and bandwidth of the BLS are critical issues as they limit the coverage distance and the maximum number of available monitoring wavelengths [44].

For the broadband source, different techniques were proposed [44]-[48]. For detection of the reflected monitoring wavelengths a series of power meters, an optical spectrum analyser (OSA) or a photodetector followed by proper signal processing and display unit as in a conventional OTDR were proposed respectively in [45], [44], and [46].

\subsection{Re-using existing light sources}

The main purpose of this approach is to avoid the use of expensive tunable OTDRs or any additional BLSs to create the test signals. Reusing downstream light sources was proposed in [49] whose principles are illustrated in Fig. 5.a. The status of upstream signals is continuously monitored. Once significant degradation in the upstream transmission quality is detected, the corresponding downstream light source is switched to transmit OTDR 
pulses instead of data. This system reacts based on upstream signals, thus can not detect the failures in drop fibers if the subscriber is off. Dynamic range of the OTDR is limited due to the fact that the peak power of the OTDR pulses should avoid crosstalk between the OTDR signal and data channels (down and upstream) [50].

\subsection{Use of commercial multi-wavelength OTDR}

The straightforward approach that one might think is the utilization of a commercial multi-wavelength OTDR which operates on all or a subset of the wavelength-grid utilized in WDM-PONs. It is clear that when compared with conventional OTDRs, such multi-wavelength OTDRs bring increased complexity and cost. Nevertheless, experimental prototypes of a so called CWDM OTDR that operate on several commonly deployed CWDM wavelengths were reported for testing applications in CWDM business access networks [5]. Fig. 5b represents the original utilization of a commercially available CWDM OTDR which can readily be adapted to a WDMPON. Operation of CWDM OTDR is more or less like the operation of a conventional OTDR. However, it is connected to a particular Mux port sending the proper wavelength. It sees the components and the behaviour of the fiber only at a particular channel's wavelength during the diagnostic of each channel [5].

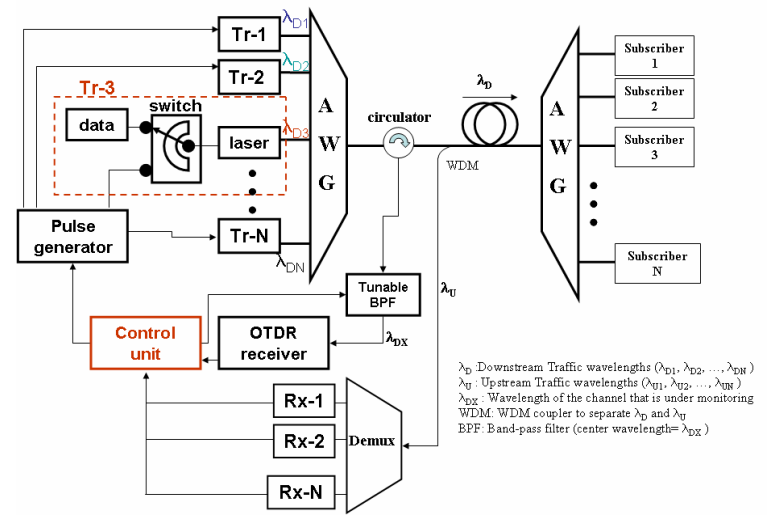

(a)

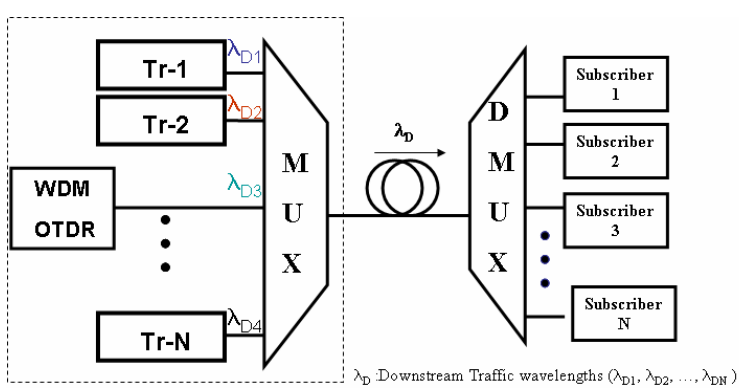

(b)

Figure 5. a) Monitoring system reusing downstream light sources [49]. Data transmission for the channel under test is interrupted, b) WDM OTDR is used to monitor channel 3 while other channels are operating [5]. Monitored downstream channel is interrupted during test.

\section{OTHER SOLUTIONS}

The Solutions based on Optical Code-division Multiplexing (OCDM) and Optical Frequency Domain Reflectometry (OFDR) techniques were reported [51], [52]. These solutions will be elaborated in the presentation.

\section{CONCLUSION}

Even though the need for monitoring the physical layer in PONs is being increasingly recognized by FTTx operators, there are no standardized methods or tools except some general recommendations in ITU-T L-series [53]. To help the development of such a document we provide a review of the existing solutions.

\section{ACKNOWLEDGEMENTS}

This work was supported in part by the FP6 ePhoton/ONE+ European Network of Excellence.

\section{REFERENCES}

[1] C.-H. Lee, Wayne V. Sorin, and B. Yoon Kim., "Fiber to the Home using a PON infrastructure", IEEE J. Lightw. Technol., vol. 24, no. 12, pp. 4568-4583, 2006.

[2] F. Effenberger, et al., "An introduction to PON technologies”, IEEE Comm. Mag., pp. S17-S25, March 2007.

[3] R.Davey, J. Kani, F. Bougart, K. McCammon, "Options for future optical access networks”, IEEE Communication Mag., pp.50-55, Oct. 2006.

[4] M. D. Vaughn, D. Kozischek, D. Meis, A. Boskovic, and R.E. Wagner, "Value of reach-and-split ratio increase in FTTH access networks", IEEE J. Lightw. Technol., vol.22, no. 11, pp. 2617-2622, 2004.

[5] N. J. Frigo, et al., "Centralized in-service OTDR testing in a CWDM business access network", IEEE J. Lightw. Technol., vol.22, no.11, pp. 2641-2652, 2004

[6] J. Lee, et al., "In-service monitoring of 16 port x 32 wavelength bi-directional WDM-PON systems with a tunable, coded optical time domain reflectometry", Optics Express, vol.15, no.11, pp. 6874-6882, May 2007.

[7] F. P. Kapron, J.D. Berardinelli, "OTDR measurements through optical splitters", Proc. of Optical Fiber Measurementss Symp., Boulder (USA), Sep. 1992, 7-10

[8] L.Wuilmart, et al., "A method for the localisation and quantization of faults in passive tree-structured optical networks using the OTDR technique", Proc. Symp. on Optical Fiber Measurements, Boulder (USA), 1996, pp. 122-123.

[9] H. Takasugi, et al., "A new fault-identification method using a dichroic reflective optical filter in optical subscriber loops", IEEE J. Lightw. Technol., vol.11, no.2, pp. 351-357, Feb. 1993.

[10] N. Tomita, et al., "Design and performance of a novel automatic fiber line testing system with OTDR for optical subscriber loops", IEEE $J$ Lightw. Technol., vol. 12, no. 5, pp. 717-726, May 1994. 
[11] N. Araki, et al., "Extended optical fiber line testing system using new eight-channel L/U band crossed optical waveguide coupler for L-band WDM transmission", IEEE J. Lightw. Technol., vol.21, no.12, pp. 316-3322, Dec. 2003.

[12] Y.-K. Chen and S.Chi, "Fault-locating and supervisory technique for multistages branched optical networks", IEEE Photonics Technol. Lett., vol.6, no.7, pp. 876-879, July 1994

[13] H. Ramanitra, et al., "Optical access network using a self-latching variable splitter remotely powered through an optical fiber link", Optical Engineering, vol. 46 (4), pp.045007/1-9, April 2007.

[14] Y. Hida et al., "Silica-based 1x32 splitter integrated with 32 WDM couplers using multilayered dielectric filters for fiber line testing at 1.65 m", IEEE J. Lightw. Technol., vol.11, no. 1, pp.96-98, Jan. 1999.

[15] K. Tanaka, M. Tateda and Y. Inoue., "Measuring the individual attenuation distribution of passive branched optical networks", IEEE Photonics Technol. Lett., vol.8, no. 7, pp. 915-917, July 1996.

[16] U. Hilbk, et al., "Selective OTDR measurements at the central office of individual fiber links in a PON", Tech. digest Optical Fiber Communication Conf., Dallas (Texas), 1997, TuK3

[17] F. Yamamoto, I. Sankawa, S. Furukawa, Y. Koyamada, and N. Takao, "In-service remote access and measurement methods for passive double star networks”, 5th Conf. Optical/Hybrid Access Networks, 1993, pp. 5.02.01-5.02.06.

[18] N. Tomita, et al., "Future targets and technologies for an optical fiber line remote testing system", Proc. Photonics Networks, Optical Technology and Infrastructure, 1997, pp.209-217.

[19] Y. Koshikiya, N. Araki, H. Izumita and F. Ito, "Simple an cost-effective fault location technique using bi-directional OTDR and in-service line testing criteria for PONs", European Conf. on Optical Commun., Glasgow, 2005.

[20] W. Chen, et al. "A novel technique for low-cost embedded non-intrusive fiber monitoring of P2MP optical access networks", in Proc. of the Optical Fiber Communication Conf., Anaheim, 2007, OThE4

[21] H. Schmuck, J. Hehmann, M. Straub and Th. Pfeiffer, "Embedded OTDR techniques for cost-efficient fibre monitoring in optical access networks", in Proc. (on CD-ROM) of the European Conf. on Optical Commun., Cannes , 2006

[22] T. Pfeiffer, et al., "Monitoring and protecting the optical layer in FTTH networks", in Proceedings of the 2005 FTTH Conf., Las Vegas, Nevada, 2005.

[23] B. De Mulder, et al., "Nonintrusive fiber monitoring of TDM optical networks", IEEE J. Lightw. Technol., vol.25, no.1, pp. 305-317, Jan. 2007.

[24] "Maintenance wavelength on fibers carrying signals", ITU-T Standardization Sector, Geneva, Switzerland, ITU-T Rec. L.41, 2000.

[25] N. Gagnon, A. Girard and M. Leblanc., "Considerations and recommendations for in-service out-of-band testing on live FTTH networks", Optical Fiber Commun. Conf., 2005, NWA3.

[26] N. Nakao, et al., "Maintenance method using 1650-nm wavelength band for optical fiber cable networks", IEEE J. Lightw. Technol., vol.19, no.10, pp. 1513-1520, 2001.

[27] F.Caviglia, et al., "Optical maintenance in PONs", European Conf. on Optical Commun., Madrid, 1998, pp. $621-626$.

[28] J. Laferriere, M. Saget, A. Champavere., "Original method for analyzing multipaths networks by OTDR measurement", Optical Fiber Commun. Conf., Dallas (Texas), 1997, pp.99-101.

[29] Y.Enomoto, H. Izumita, M. Nakamura., "Over $31.5 \mathrm{~dB}$ DR optical fiber line testing system with optical fiber fault isolation function for 32-branched PON", OFC Conf., Atlanta, 2003, pp.608-609.

[30] N. Araki, Y. Enomoto, "Improvement of fault identification performance using neural networks in passive double star optical networks", Optical Fiber Commun. Conf., San Jose, 1998, pp. 223-224.

[31] N.Araki, H. Izumita, Y. Koshikiya, M. Nakamura, "High spatial resolution PON measurement using an OTDR enhanced with a dead-zone-free signal analysis method", Symp. on Optical Fiber Measurements, 2004, pp.69-72.

[32] K. Ozawa, et al., "Field trial of in-service individual line monitoring of PONs using a tunable OTDR", 14th Int. Conf. on Optical fiber Sensors, Venice, 2000, pp.880-883.

[33] C.-K. Chan et al., "A practical passive surveillance scheme for optically amplified passive branched optical networks", IEEE Photonics Technol. Lett., vol.9, no.4, pp. 526-528, 1997.

[34] C.-K. Chan, F. Tong, L.-K. Chen, K.-P. Ho and D. Lam, "Fiber-fault identification for branched access networks using a wavelength-sweeping monitoring source", IEEE Photonics Technol. Lett., vol.11, no.5, pp. 614-616, May 1999.

[35] C.-H. Yeh and S. Chi, "Optical fiber-fault surveillance for PONs in S-band operation window", Optics Express, vol.13, no. 14, 5494-5498, July 2005.

[36] C.-H. Yeh and S. Chi, "Fiber-fault monitoring technique for PONs based on fiber Bragg gratings and semiconductor optical amplifier", Optics Commun., vol.257, pp.306-310, 2006.

[37] Y. Li, D. Wang, and J. Li., "FTTH remote fiber monitoring using optical wavelength domain reflectometry (OWDR) and wavelength coded tag (WCT)", Optical Fiber Commun. Conf., 2006, OThU3.

[38] K. Yuksel, S. Dupont, L. Robette, D. Hamoir, J.-C. Froidure, "OTDR-based fault surveillance method for tree-structured PONs", Proc. of the 15th Int. Symp. on Services and Local Access (on CD-ROM), Edinburg, 2004.

[39] K. Yuksel, S. Dupont, D. Hamoir, J.-C. Froidure, "FTTx automated test solution: requirements and experimental implementation", Electronics Lett., vol. 41, no. 9, pp.546-547, April 2005.

[40] K. Yuksel, et al., "Centralised Optical Monitoring of Tree-structured PONs using a Raman-assisted OTDR”, in Proc. (on CD-ROM) of Int. Conf. on Transparent Optical Networks, Rome, 2007.

[41] N. Honda, D. Iida, H. Izumita and F. Ito, "Bending and connection loss measurement of PON branching fibers with individually assigned Brillouin frequency shifts", Optical Fiber Commun. Conf., Anaheim (California), 2005, OThP6.

[42] D. Iida, N. Honda, H. Izumita and F. Ito, "Design of identification fibers with individually assigned BFS for monitoring PONs", IEEE J. Lightw. Technol., vol.25, vol. 5, pp. 1290-1297, May 2007.

[43] S. Hann, J.-S. Yoo and C.-S. Park, "Monitoring technique for a hybrid PS/WDM-PON by using tunable OTDR and FBGs", Meas. Sci. Technol., vol.17, pp. 1070-1074, 2006 .

[44] J. H. Lee, Y.-G. Han, and S. B. Lee, "WDM-PON architecture with C-band OLT, L-band ONU, and U-band monitoring based on FP-LDs wavelength-locked by a single, depolarized, CW supercontinuum", Optical Fiber Commun. Conf., Anaheim (Californie), 2007, OTuG3

[45] S.-B. Park, et al., "Optical fault monitoring method using broadband light source in WDM-PON", Electronics Lett., vol. 42, no. 4, pp. 239-241, Feb. 2006.

[46] J. Park, J. Baik, and C. Lee, "Fault-detection technique in a WDM-PONs", Optics Express, vol. 15, no. 4, pp. 1461-1466, 2007.

[47] K. Lee, et al., "Fiber link loss monitoring scheme in bidirectional WDM transmission using ASE-injected FP-LD", IEEE Photonics Technol. Lett., vol.18, no. 3, pp. 523-525, Feb. 2006.

[48] E. Wong, X. Zhao and C. J. Chang-Hasnain, "Novel fault monitoring and localization scheme in WDM-PON with upstream VCSEL transmitter", Optical Fiber Commun. Conf., Anaheim, 2007, OthE3.

[49] K. W. Lim, et al., "Fault localization in WDM passive optical network by reusing downstream light sources", IEEE Photonics Technol. Lett., vol. 17, no.12, pp. 2691-2693, Dec. 2005.

[50] E.S. Son, et al., "Localization of fiber failures in WDM-PON by reusing downstream light sources", Optical Fiber Commun. Conf., 2006.

[51] H. Fathallah, L.A. Rusch, "Code-division multiplexing for in-service out-of-band monitoring of live FTTH-PONs", J. of Optical Networking, vol.6, no.7, pp. 819-829, July 2007.

[52] N. Zou, Y. Nmihira, C. Ndiaye, H. Ito, "Fault location for branched optical fiber networks based on OFDR technique using FSF laser as light source", Optical Fiber Commun. Conf., 2006, NWC2.

[53] "Optical fibre maintenance criteria for access networks", ITU-T Standardization Sector, Geneva, Switzerland, ITU-T Recommendation L.53. 